\title{
An Effective Method of Secret-Fragment- Visible Mosaic Video Generation Using HAAR Wavelet Transformation
}

\author{
Swapnil Patil ${ }^{1}$, A. A. Deshmukh ${ }^{2}$ \\ ${ }^{1}$ Department of ENTC Engineering G. H. Raisoni Institute Engineering \& Technology, Pune, India \\ ${ }^{2}$ Professor, Department of ENTC Engineering, G. H. Raisoni Institute Engineering \& Technology, Pune, India
}

\begin{abstract}
In this paper present a method for creating a video mosaic, a two dimensional arrangement of small source videos (tiles) that suggests a larger, unified target video. The mosaic video is the video which looks similar to arbitrarily selected target video is yielded by dividing the secret video. In the existing system work on the mosaic images is already done by number of researcher using different methods. Now here we are working on videos to make it mosaic by using HARR Wavelet Transform. A method for creating a video mosaic by using HARR Wavelet Transform consist of, a two dimensional arrangement of small source videos (tiles) that suggests a larger, unified target video. In this paper, we have suggested the transform domain method for digital video watermarking for embedding invisible watermarks behind the video. It is used for copyright protection as well as proof of ownership. In this paper, we first extracted the frames from the video and then used frequency domain characteristics of the frames for watermarking. In this paper, we have specifically used the characteristics of the Discrete Cosine Transform for watermarking and calculated different parameters. We also introduce a dynamic programming algorithm that automatically chooses the smaller tiling sub-sequences from a large collection of candidate source video sequences to best match the target video. After the selection process, the color in the tiling videos is automatically adjusted to better suggest the target video. Finally, our method also supports the use of different tiling shapes. The information required for recovering the secret image is embedded into the created mosaic video by a lossless data hiding scheme using a key. Good experimental results show the feasibility of the proposed Method.
\end{abstract}

Keywords: Data Hiding, Mosaic, Still Images, Watermarking etc

\section{Introduction}

In today's life providing security has become more cumbersome because of all the malicious possibilities in data transmission, so we need a system which makes data transmission more A new method of combining art image generation and hiding a secret image into this cubism like image to enhance the camouflage effect for various information-hiding applications is proposed. First, a new type of computer art, called line-based Cubism-like image, which keeps a characteristic of the cubism art created by extract prominent lines and regions[1]. For creating the mosaic video we need two video named as "source" and "target" audio files. The first process is picking the target and the source audio for mosaic creation. Picking of target audio is similar to that of the selected source audio but not the same. Then converting the lager source video into several small video for secret purpose. The splitting is used to placed the source video into target video. after that using the separate algorithm to find the most similar part of target image for placing the source tile video. This would be done for all the split source video. Then we are composing these audio in a mosaic form for hiding the secret information[1][3]. The mosaic video is same as that of the target audio but this contain the tile source audio not hear. The output video will send to the destination, in destination re-mosaic process is held based on the some algorithm. The tile images are retrieving first for constructing the source image as send by the sender. After that the tile images are combined to create a original Video signal is basically any sequence of time varying images[2]. A still image is a spatial distribution of intensities that remain constant with time, whereas a time varying image has a spatial intensity distribution that varies with time. Video signal is treated as a series of images called frames. An illusion of continuous video is obtained by changing the frames in a faster manner which is generally termed as frame rate.

\section{Literature Survey}

In the proposed system initially the source image is converted into Cubism-like-art image by extracting prominent lines and regions. Yi-Zhe Song, Paul L. Rosin, Peter M. Hall and John Collomosse [3] proposed a method to simple shapes (e.g. circles, triangles, squares, super ellipses and so on) are optimally fitted to each region within a segmented photograph. Stipple Placement using Distance in a Weighted Graph is proposed by David Mould [4] provides extra emphasis to image features, especially edges. Regarding lossless data hiding, several techniques have been proposed. Xiaomei Quan and Hongbin Zhang proposed "Lossless Data Hiding Scheme Based On Lsb Matching [4] deals data hiding based on bit change. A lossless data hiding method based on histogram shifting and encryption is proposed by Nutan Palshikar and Prof. Sanjay Jadhav, and C. Liu in Lossless Data Hiding using Histogram Modification and Hash Encryption Scheme [5] A novel scheme for separable reversible data hiding in encrypted images developed by Nutan Palshikar, Prof. Sanjay Jadhav in Separable Reversible Data Hiding in Encrypted Image [6]. A new secure image transmission technique which automatically transforms a given large-volume secret image into a so-called secret-fragment visible mosaic image of the same size [7]. 


\section{International Journal of Science and Research (IJSR) \\ ISSN (Online): 2319-7064}

Index Copernicus Value (2013): 6.14 | Impact Factor (2014): 5.611

\section{Existing Architecture}

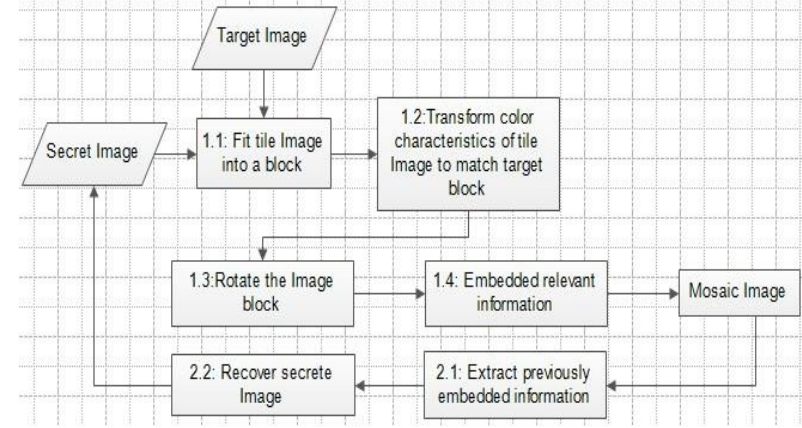

Figure 1: Existing Architecture

Phase 1-creation of a secret-fragment-visible mosaic image using the tile images of a secret image and the selected similar target image as input.

Phase 2 -recovery of the secret image from the created secret-fragment-visible mosaic image. The first phase includes three stages of operations.

Stage 1.1 -searching a target image most similar to the secret image.

Stage 1.2 -fitting the tile images in the secret image into the blocks of the target image.

Stage 1.3 -create a blank image to create a mosaic Image. Stage 1.4-embedding the tile-image fitting information into the mosaic image for later secret image recovery. And the second phase includes two stages of operations:

Stage 2.1 -retrieving the previously-embedded tile image fitting information from the mosaic image.

Stage 2.2-reconstructing the secret image from the mosaic image using the retrieved information.

Proposed methodology has been divided into 2 phases.

\section{Mosaic Image Creation}

Fig 1 shows In this first phase, Shamir secret sharing algorithm is used by which a secret is divided into parts, giving each participants its own unique part, some of the parts or all of them are needed in order to reconstruct the secret counting on all participants to combine together, the secret might be impractical and therefore sometimes the threshold scheme is used. Now fitting the tile images of the secret image into the target blocks of a preselected target image. After this transforming the color characteristic of each tile image in the secret image to become that of the corresponding target block in the target image and rotating each tile image into a direction with the minimum RMSE value with respect to its corresponding target block. After the rotation embedding relevant information into the created mosaic image for future recovery of the secret image. In this way we get the output secret fragment visible mosaic image.

\section{Secret Image Recovery}

In this second phase, extracting the embedded information for secret image recovery from the mosaic image, and recovering the secret image using the extracted information by secret image recovery algorithm. In this phase result will be calculated and optimize if required result is in the form of delay and accuracy

\section{Existing System}

In traditional methods secret text can be hidden into image which is called as Steganography. In this method only text data can be encrypted but not image. Secret images can be hidden using water marking principles. Water marking is very simple process and it is weak that anyone can decrypt easily. Mosaic image technique is one of the efficient techniques to hide the secret images. This methodology needs another image which is said to be cover image. Creating mosaic image is also a art of computer. In Existing steganography techniques may be classified into three categories - image, video, and text steganography, and image steganography aims to embed a secret message into a cover image with the yielded stego-image looking like the original cover image. Many image steganography techniques have been proposed [1-4], and some of them try to hide secret images behind other images [3-4]. The main issue in these techniques is the difficulty to hide a huge amount of image data into the cover image without causing intolerable distortions in the stego-image.

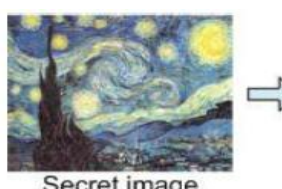

Secret image

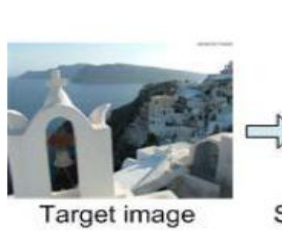

\section{Existing Model (Working On Images)}

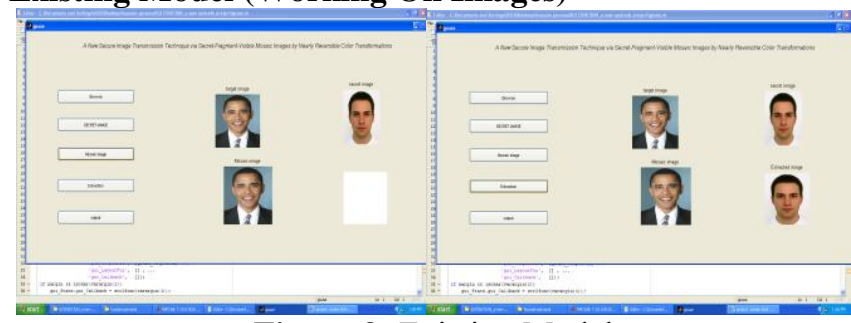

Figure 3: Existing Model

\section{Proposed Methodology}

\section{Frame Type}

Three types of video frames are I-frame, P-frame and Bframe. „' ${ }^{\text {ee }}$ stands for Intra coded frame, ,P ${ }^{\text {ee }}$ stands for Predictive frame and , $\mathrm{B}^{\mathrm{ec}}$ stands for Bidirectional predictive frame. „I" frames are encoded without any motion compensation and are used as a reference for future predicted „, $\mathrm{P}^{\mathrm{ee}}$ and , $\mathrm{B}^{\mathrm{ec}}$ type frames. „I $\mathrm{I}^{\mathrm{e}}$ frames however require a relatively large number of bits for encoding. , $\mathrm{P}^{\mathrm{ec}}$ frames are encoded using motion compensated prediction from a reference frame which can be either , $\mathrm{I}^{\mathrm{ee}}$ or , $\mathrm{P}^{\mathrm{ee}}$ frame. „, $\mathrm{P}^{\mathrm{ec}}$ frames are more efficient in terms of number of bits

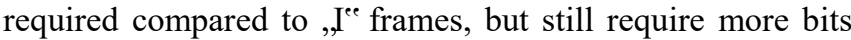
than , $\mathrm{B}^{\text {ce }}$ frames. , $\mathrm{B}^{\mathrm{ce}}$ frames require the lowest number of bits compared to both, „Ie and „P, $\mathrm{P}^{\mathrm{Ie}}$ frames but incur computational complexity. Frames between two successive 


\section{International Journal of Science and Research (IJSR) \\ ISSN (Online): 2319-7064}

Index Copernicus Value (2013): 6.14 | Impact Factor (2014): 5.611

„I' frames, including the leading ,I' frame, are collectively called as group of pictures (GOP). The GOP is illustrated in figure 4 . The illustrated figure has one ,I $\mathrm{I}^{\mathrm{ee}}$ frame, two „P, frames and six , $\mathrm{B}^{\mathrm{ee}}$ frames. Typically, multiple , $\mathrm{B}^{\mathrm{ee}}$ frames are inserted between two consecutive , $\mathrm{P}^{\mathrm{ee}}$ or between, $\mathrm{I}^{\mathrm{ee}}$ and ,Pe frames. The existence of GOPs facilitates the implementation of features such as random access, fast forward or fast and normal reverse playback

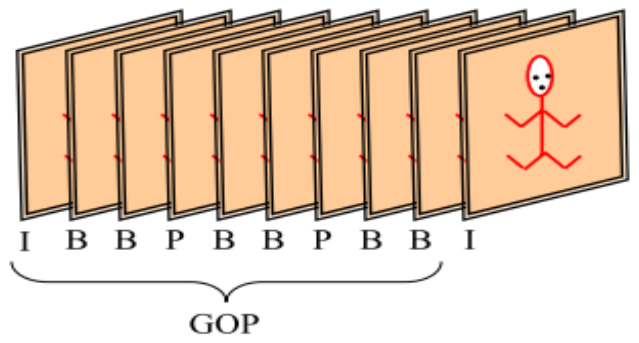

Figure 4: Group of Picture

\section{Video Processing}

Video processing technology has revolutionized the world of multimedia with products such as Digital Versatile Disk (DVD), the Digital Satellite System (DSS), high definition television (HDTV), digital still and video cameras. The different areas of video processing includes (i) Video Compression (ii) Video Indexing (iii) Video Segmentation (iv) Video tracking etc.

\section{Haar Wavelet Transformation}

Image processing and analysis based on the continuous or discrete image transforms are classic techniques. The image transforms are widely used in image filtering, data description, etc. Now a days the wavelet theorems make up very popular methods of image processing, de noising and compression. Considering that the HAAR functions are the simplest wavelets, these forms are used in many methods of discrete image transforms and processing. The image transform theory is a well known area characterized by a precise mathematical background, but in many cases some transforms have particular properties which are not still investigated. This paper for the first time presents graphic dependences between parts of HAAR and wavelets spectra. It also presents a method of image analysis by means of the wavelets-HAAR spectrum. Some properties of the HAAR and wavelets spectrum were investigated. The extraction of image features immediately from spectral coefficients distribution were shown. In this paper it is presented that two-dimensional both, the HAAR and wavelets functions products man be treated as extractors of particular image features[12].

\section{Digital Watermarking}

Digital watermarking includes a number of techniques that are used to imperceptibly convey information by embedding it into the cover data. Here the cover data taken is a video sequence and watermarking is thus called Video Watermarking. Video watermarking is a field that is rapidly evolving in the area of multimedia and interest of the people in this field is increasing day. Major Factor

1) Privacy of the digital data is required and copying of a video is comparatively very easy.

2) Fighting against the "Intellectual property rights breach".

3) Tempering of the digital video must be concealed.

4) Copyright protection must not be eroded.

\section{Transform Domain Watermarking}

A watermarking algorithm using transform domain techniques focus on embedding information in the frequency domain of the video as opposed to the spatial domain. The most popular transforms, where the frequency domain watermarking algorithms work, are Discrete Fourier Transform (DFT), Discrete Cosine Transform (DCT) and Discrete Wavelet Transform (DWT). These are applied to transform a frame of the video into the frequency domain where the coefficients of the digital frame are separated into different priorities in accordance with the human perception system. The watermark bits are embedded by modulating the magnitude of these coefficients. Watermarking in the transform domain is applied in three steps as explained below.

1) Forward Transform: The frame of the video is first transformed to a domain that facilitates data embedding.

2) Embedding: The subset of the transform coefficients is modified with the prepared signature data. One can employ a model of human perception to weigh the strength of the embedding modifications. By choosing a suitable frequency transform domain and selecting only certain coefficients (low- to mid frequency range), a lot of human visual system (HVS) modeling can be done implicitly. The better the transform approximates the properties of HVS, the easier it is to put more energy in the embedded signal without causing perceptible distortion.

3) Inverse Transform: The modified coefficients are inversely transformed to produce a watermarked frame.

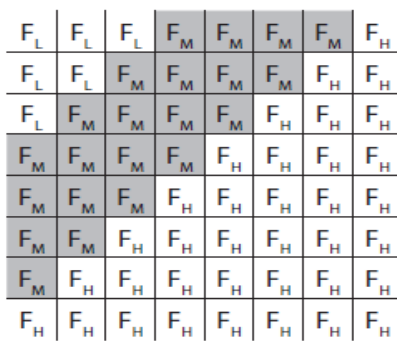

(a)

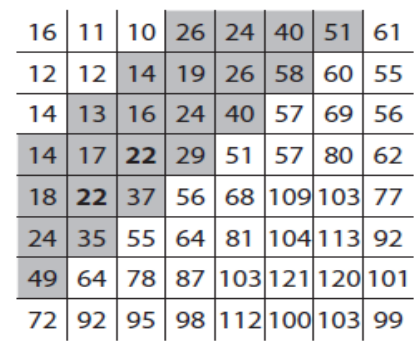

(b)

Figure 5: Definition of DCT regions and Quantization values used in JPEG compression scheme

Figure 5(a) shows the three regions in the frequency domain. FL is used to denote the lowest frequency components of the block, while FH is used to denote the higher frequency components. FM is chosen as the embedding region as to provide additional resistance to lossy compression techniques, while avoiding significant modification of the cover video. 


\section{International Journal of Science and Research (IJSR) \\ ISSN (Online): 2319-7064}

Index Copernicus Value (2013): 6.14 | Impact Factor (2014): 5.611

\section{Discrete Cosine Transform}

Discrete cosine transformation [2, 3, 4] (DCT) transforms a signal from the spatial into the frequency domain by using the cosine waveform. DCT concentrates the information energy in the bands with low frequency, and therefore shows its popularity in digital watermarking techniques. The DCT allows a frame to be broken up into different frequency bands, making it much easier to embed watermarking information into the middle frequency bands of a frame. The middle frequency bands are chosen such that they have minimized to avoid the most visual important parts of the frame (low frequencies) without over-exposing themselves to removal through compression and noise attacks (high frequencies).

Two-dimensional DCT of a frame with size $\mathrm{M} \times \mathrm{N}$ and its inverse DCT (IDCT) are defined in Equations 1 and 2, respectively.

Two-dimensional DCT of a frame with size $\mathrm{M} \times \mathrm{N}$ and its inverse DCT (IDCT) are defined in Equations 1 and 2, respectively.

$$
\begin{aligned}
& F(u, v)=\alpha(u) \alpha(v) \sum_{y=0}^{M-1} \square \sum_{y=0}^{N-1} f(x, y) \\
& \cos \left[\frac{(2 x+1) u \cdot \pi}{2 \cdot M}\right] \cos \left[\frac{(2 y+1) v \cdot \pi}{2 \cdot N}\right]
\end{aligned}
$$

where

$$
\begin{array}{ll}
\alpha(u)=\sqrt{1 / M} & \text { for } \mathrm{u}=0 ; \\
\alpha(u)=\sqrt{2 / M} & \text { for } \mathrm{u}=1,2,3 \ldots \mathrm{M}-1 ; \\
\alpha(v)=\sqrt{1 / N} & \text { for } \mathrm{v}=0 ; \\
\alpha(v)=\sqrt{2 / N} & \text { for } \mathrm{v}=1,2,3, \ldots \mathrm{N}-1 ; \\
f(x, y)=\sum_{v=0}^{N-1} \alpha(u) \alpha(v) F(u, v) & \\
\cos \left[\frac{(2 x+1) u \cdot \pi}{2 \cdot M}\right] \cos \left[\frac{(2 y+1) v \cdot \pi}{2 \cdot N}\right]
\end{array}
$$

where $x=0,1,2, \ldots M-1, y=0,1,2, \ldots N-1$

\section{Idea of Watermarking}

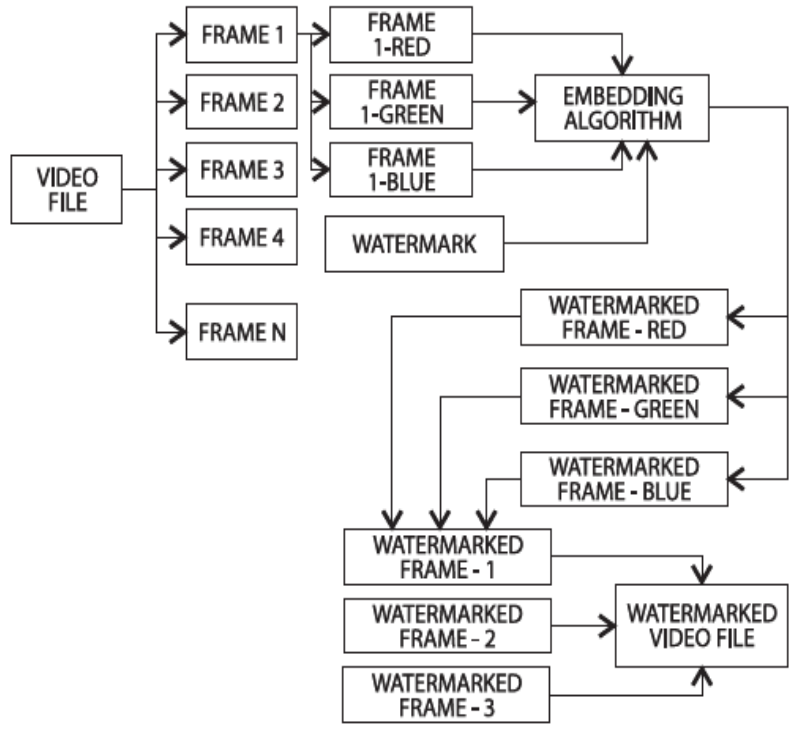

Figure 6: Watermarking Process
Fig 6 shows the idea of video watermarking at the sending end. Here, first of all frames is extracted from the video sequence. The next step is to divide the frame into its Red, Green and Blue part. Each part is then individually given to the embedding algorithm block where the other input is a watermark that is to be embedded. After each part is watermarked, the next frame is taken and the procedure is repeated until the last frame. After the watermark is embedded in every frame, all frames are mixed to make the watermarked video which is then transmitted in the channel.

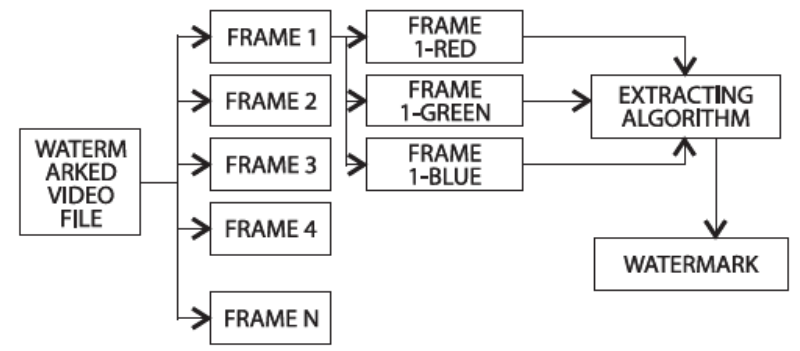

Figure 7: Extracting Watermarking Process

Figure 7 shows the idea of video watermarking at the receiver end. Here, a watermarked video is divided into the frames which are divided into the Red, Green and Blue part from where the watermark is extracted. This Procedure is repeated for all frames so as to recover the watermark.

$$
\begin{aligned}
& M S E=\frac{1}{M \cdot N} \sum_{x=1}^{M} \sum_{y=1}^{N}\left\{\left(f(x, y)-f^{4}(x, y)\right)^{2}\right\} \\
& \text { PSRN }=10 \cdot \log \frac{255^{2}}{M S E},
\end{aligned}
$$

Where

MSE - Mean Square Error, PSNR - Peak Signal to Noise Ratio,

$\mathrm{f}(\mathrm{x}, \mathrm{y})$ - Original Frame of the Video,

$\mathrm{f}^{\mathrm{e}}(\mathrm{x}, \mathrm{y})$ - Watermarked Frame of the Video.

The phrase peak signal-to-noise ratio, often abbreviated as PSNR, is an engineering term for the ratio between the maximum possible power of a signal and the power of corrupting noise that affects the fidelity of its representation. Because many signals have a very wide dynamic range, PSNR is usually expressed in terms of the logarithmic decibel scale. A higher PSNR would normally indicate that the reconstruction is of higher quality at the receiver end. PSNR is most easily defined via the Mean Square Error (MSE) which holds for two $m \times n$ Frames $f$ and $f$ ' where one of the Frames is considered a noisy approximation of the other.

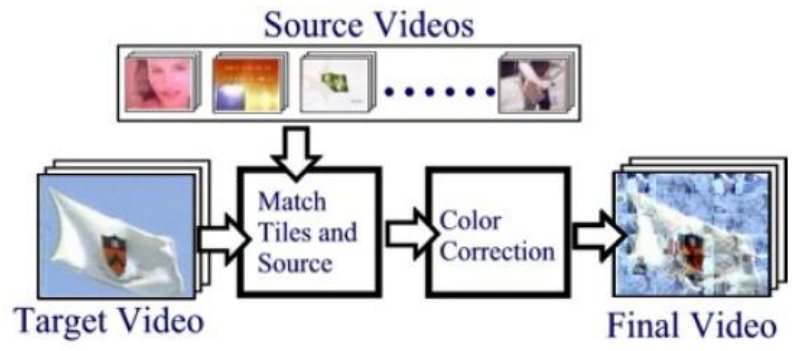

Figure 8: Mosaic Video Creation 


\section{International Journal of Science and Research (IJSR) \\ ISSN (Online): 2319-7064}

Index Copernicus Value (2013): 6.14 | Impact Factor (2014): 5.611

\section{Steps Creating Mosaic Video}

Fig 7,Fig 8 creating a video mosaic involves the following steps:

1. Select a target video, $T$.

2. Select a corpus of video sources, $S$

3. Select a tiling structure. This may be a simple as an $n \times n$ grid of rectangles or a more complex tiling pattern.

4. For each tile of the target video:

(a) For each frame of the target video: i. Select the best frame $S j$ from the source videos matching the current tile in the current frame of $T$.

ii. Color correct $S j$ to mimic the current tile in the current frame of $T$.

iii. Paste $S j$ into the tile for output.

\section{Proposed Architecture}

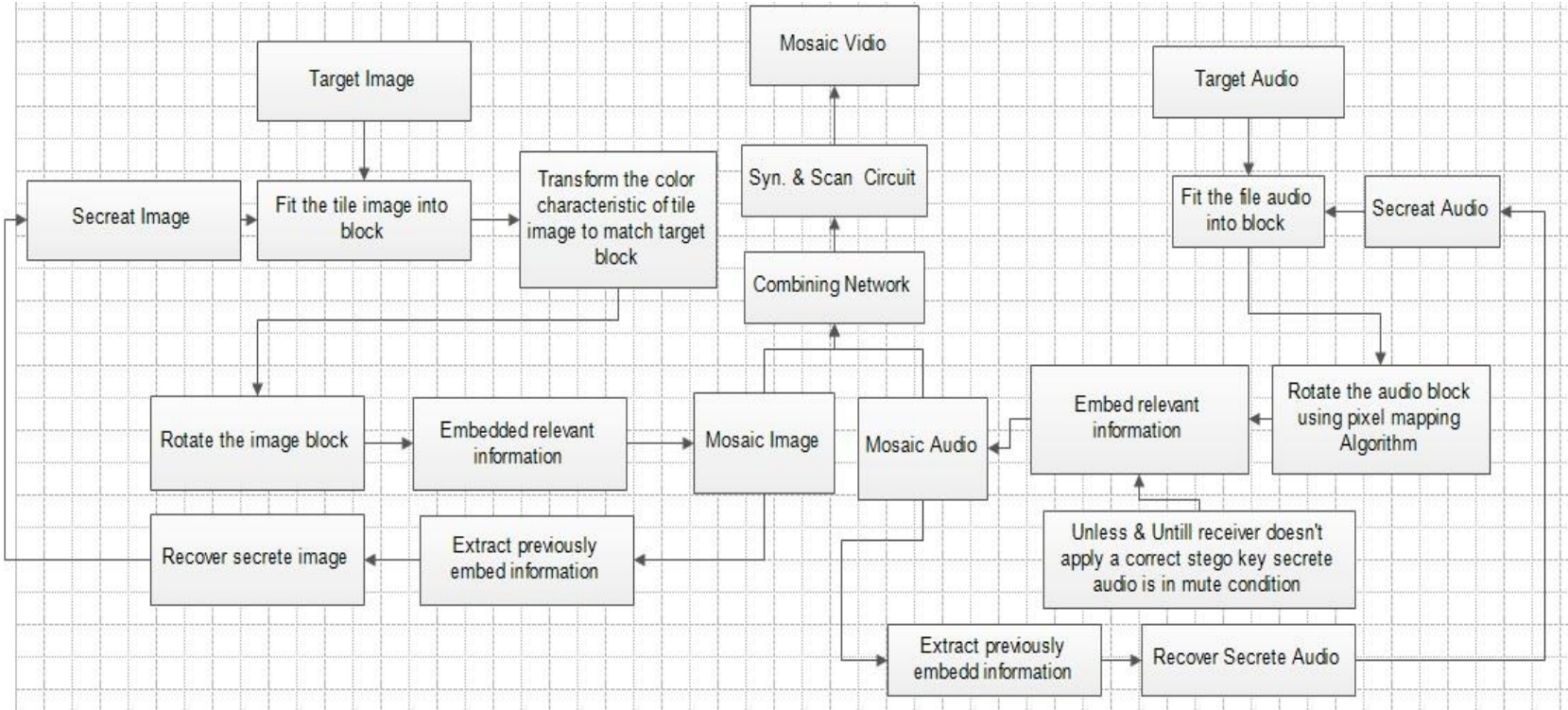

Figure 8: Proposed Mosaic video Architecture

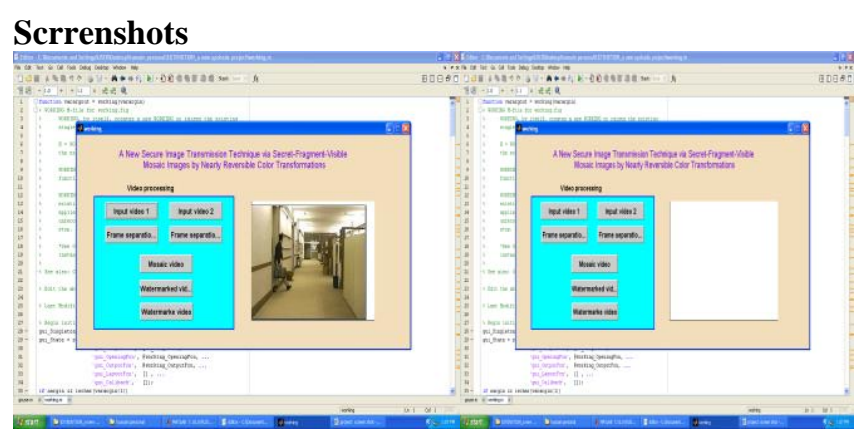

Hardware Requirements

- Processor i3 GHz.

- Hard Disk 500 GB.

- RAM :2 GB

\section{Software Requirement}

- Operating system : Win 7,8

- Coding Language : MATLAB

- Tool :R 200

\section{Conclusion}

We have presented a system for creating video mosaics an arrangement of small videos that suggests a larger, unified video sequence. We solve the main challenge of providing both visual matches and temporal stability in a computationally efficient way, while still being flexible enough to accommodate a variety of matching criteria.
There are many more possibilities for altering the final look of the video mosaic:

Different tile shapes and animated tiles: There is no reason the tiles need to be simple rectangles. In fact, if one segments the target video into semantically meaningful regions, the tiles shapes could be modified to follow these region boundaries.

Allowing temporal distortions: Tiling sequences to be temporally distorted (slowed down or sped up) to achieve better matches. Finding the best temporal distortions presents an interesting problem.

Different matching criteria: Doubtless, there are other matching criteria worth investigating. Given a large enough corpus of source video, one might also score the match based on semantic information.

Incorporating video textures: Another way of collecting good tiling footage may be to synthesize longer sequences from shorter ones using the video textures framework

\section{References}

[1] Nutan Palshikar, Prof. Sanjay Jadhav, "Lossless Data Hiding using Histogram Modification and Hash Encryption Scheme ", in International Journal of Emerging Technology and Advanced Engineering,January 2014. 


\section{International Journal of Science and Research (IJSR) \\ ISSN (Online): 2319-7064}

Index Copernicus Value (2013): 6.14 | Impact Factor (2014): 5.611

[2] Swapnil patil, A.A Deshmukh" A Review On Modern Secure Mosaic Video Generation For Secure Video Generation" International Journal Of Emerging Trends And Technology In Computer Science.(IJETTCS)ISSN2278-6856 Volume 4 Issue 2

[3] Xinpeng Zhang, "Separable Reversible Data Hiding in Encrypted Image", in in Proc. IEEE Trans. on Information Forensics and security,Vol. 7, No. 2, APRIL 2012.

[4] Ya-Lin Lee and Wen-Hsiang Tsai, "A new secure image transmission technique via secret- fragmentvisible mosaic images by Nearly-reversible Color Transformations," in IEEE Trans. 2013.

[5] Wei-Jen Wang, Cheng-Ta Huang, and Shiuh-Jeng Wang ,"VQ Applications in Steganographic Data Hiding Upon Multimedia Images" IEEE Systems Journal, Vol. 5, No. 4, December 2011.

[6] Kotaro Yamamoto , "Real-Time Audio Watermarking Based on Characteristics of PCM in Digital Instrument," in Journal of Information Hiding and Multimedia Signal Processing ,2010

[7] Hussein A. Aly, "Data Hiding in Motion Vectors of Compressed Video Based on Their Associated Prediction Error," IEEE Trans On Information Forensics And Security, Vol. 6, No. 1, March 2011.

[8] Ersin Esen and A. Aydin Alatan ,"Robust Video Data Hiding Using Forbidden Zone Data Hiding and Selective Embedding," in Trans On Circuits And Systems For Video Technology, Vol. 21, No. 8, August 2011 .

[9] Zahra Toony and Mansour Jamzad,"A Novel Image Hiding Scheme Using Content Aware Seam Carving Method" in International Conference on Availability, Reliability and Securityy, 2010.

[10] V.Rajkumar ," Modifier Digital Image Steganography Using Discrete Wavelet Transform,", in Trans On Circuits And Systems For Video Technology,Volume 1, Issue 1, March 2013 Nilanjan Dey, Anamitra Bardhan Roy and Sayantan Dey ,"A Novel Approach of Color Image Hiding using RGB Color planes and DWT ," in International Journal of Computer Applications (0975 8887), Volume 36- No.5, December 2011.

[11]Zeng L., Jansen C. P., Marsch S., Unser M., Hunziker Four-Dimensional Wavelet Compression of Arbitrarily Sized Echocardiographic Data. IEEE Transactions on Medical Imaging, 21(9), 1179-1188.2003

[12] Drori I., Lischinski D.: Fast Multi resolution Image Operations in the Wavelet Domain. IEEE Transactions on Visualization and Computer Graphics, 9(3), 395411.

[13]Fernandes F., Selesnick I., van Spaendonck R., Burrus C.: ComplexWavelet Transforms with Allpass Filters. Signal Processing, 83(8), 1689-1706. 